\title{
ANÁLISIS DE LA CONTAMINACIÓN AMBIENTAL PRODUCIDA POR EL PLASMA FRÍO DE BAJA PRESIÓN EN LA LIMPIEZA DE LÁMINAS METÁLICAS
}

\author{
Environmental Pollution Analysis \\ Produced By Low-Pressure Cold \\ Plasma in the Sheet Metal Cleaning \\ PROCESS
}

\author{
Luis M. López ${ }^{1, *}$, Janine Villavicencio Ramírez ${ }^{1}$, \\ Kevin Aponte ${ }^{2}$, Jorge I. Fajardo ${ }^{2}$, Christian P. López ${ }^{3}$
}

\section{Resumen}

La presente investigación aborda un análisis del nivel de contaminación producido por los gases generados de monóxido de carbono $(\mathrm{CO})$, dióxido de carbono $\left(\mathrm{CO}_{2}\right)$ y los hidrocarburos $(\mathrm{HC})$ en la limpieza de láminas metálicas de acero inoxidable AISI/SAE 304 aplicando plasma frío de oxígeno a baja presión para la remoción de los aceites ISO 32, ISO 68 e ISO 220, con diferentes parámetros de control del generador de acuerdo con el aceite lubricante removido de la superficie de la lámina metálica de acero inoxidable. La experimentación se realizó en un primer proceso con una descarga aplicada directamente a la superficie de la lámina impregnada con el aceite colocando con un volumen de $0,1 \mathrm{ml}$ y en un segundo proceso donde la lámina con el aceite impregnado fue sumergida en un desengrasante para aceites y grasas con la finalidad de realizar una limpieza previa a la aplicación del plasma frío a baja presión en la superficie. Para el análisis de los resultados en el nivel de gases generados por cada aceite se aplica un análisis estadístico para determinar si existe una diferencia significativa en el nivel de los gases generados en las dos etapas.

Palabras clave: plasma frío a baja presión, contaminación

\section{Abstract}

The present research addresses an analysis of the level of contamination produced by gases generated from carbon monoxide $(\mathrm{CO})$, carbon dioxide $(\mathrm{CO} 2)$ and hydrocarbons $(\mathrm{HC})$ in the cleaning of metallic sheets of stainless steel AISI / SAE 304, when applying low pressure cold oxygen plasma for the removal of oils ISO 32, ISO 68 and ISO 220, using different generator control parameters according to the lubricating oil removed from the surface of the stainless steel metallic sheet. The experimentation was carried out in a first phase in which a discharge was applied directly to the surface of the sheet contaminated with a volume of $0.1 \mathrm{ml}$ of oil, and in a second phase in which the sheet with the oil was immersed in an oil degreaser to perform a pre-cleaning prior to the application of low pressure cold plasma on the surface. For analyzing the results in the level of gases generated by each oil, a statistical analysis is applied to determine if there is a significant difference in the level of the gases generated between the two phases.

Keywords: Low pressure cold plasma, contamination.

\footnotetext{
1,* Universidad Nacional Mayor de San Marcos, Perú. Autor para correspondencia $₫ 1 l o p e z @ u p s . e d u . e c$.

(D) http://orcid.org/0000-0001-9353-384X, (D) http://orcid.org/0000-0001-8831-2604

${ }^{2}$ Grupo de Investigación GiMaT, Universidad Politécnica Salesiana, Ecuador. (D http://orcid.org/0000-0003-0359-1750, (D) http://orcid.org/0000-0003-1047-398X

${ }^{3}$ Vaughn College of Aeronautics and Technology, New York, USA. (D) http://orcid.org/0000-0002-0211-4613
}

Recibido: 02-04-2019, aprobado tras revisión: 06-05-2019

Forma sugerida de citación: López, L. M.; Villavicencio Ramírez, J.; Aponte, K.; Fajardo, J. I. y López, C. P. (2019). «Análisis de la contaminación ambiental producida por el plasma frío de baja presión en la limpieza de láminas metálicas». INGENIUS. N. ${ }^{\circ} 22$, (julio-diciembre). pp. 17-24. DOI: https://doi.org/10.17163/ings.n22.2019.02. 


\section{Introducción}

Dentro de los procesos productivos se busca la eliminación o reducción de emisiones, vertimientos y desechos con el uso eficiente de los recursos, con tecnologías que permitan alcanzar los requerimientos y especificaciones de un producto con el menor impacto ambiental [1].

La contaminación producida por los baños de decapado en la limpieza de láminas metálicas con ácidos y bases, considerados tóxicos, nocivos y peligrosos para la salud, infraestructuras y medioambiente se ha incrementado considerablemente en estos últimos años. Los metales pesados como zinc, cromo y cobre que se acumulan en los baños de decapado se consideran teóricamente como sustancias suspendidas y son considerados como otra problemática por la grave contaminación ambiental que producen [2-5].

En la actualidad se desarrollan diversos procesos de limpieza superficial que buscan reducir o eliminar la contaminación como nuevas alternativas a procesos tradicionales con la misma eficiencia, considerando que la presencia de impurezas o restos de aceites y grasas en las diferentes superficies a limpiar restará la adherencia en tratamientos superficiales posteriores $[6,7]$.

La aplicación del plasma frío a baja presión según estudios previos presenta resultados satisfactorios en la remoción de aceites en láminas metálicas, utilizando gases no contaminantes, con la finalidad de remover tanto compuestos mecánicos como orgánicos [8]. De la eficacia de este tratamiento dependerán las propiedades adhesivas del material según el ángulo de contacto que está directamente relacionado con la energía libre superficial [9-11].

La limpieza superficial de componentes orgánicos por plasma frío a baja presión es un método que mediante el bombardeo de iones de un determinado gas generado por efectos físicos o reacciones químicas transforma a las sustancias que se encuentran sobre las láminas metálicas en su fase gaseosa que son expulsadas de la cámara hacia la atmósfera [12]. En investigaciones realizadas por $[6,13]$ se indica que la aplicación de plasmas fríos en ciertos procesos industriales se realizan de forma más eficiente y barata, con lo que se reduce la contaminación y los residuos tóxicos generados. El proceso de limpieza presenta una mayor eficiencia en la calidad de la limpieza al disminuir el ángulo de contacto cuando se realiza una inmersión previa en un desengrasante [8] Figura 1.

El ángulo de contacto es una propiedad superficial de los sólidos que cuantifica su tendencia a la hidrofobicidad como parámetro importante para analizar los mecanismos de interacción entre las fases sólida y líquida que se presentan en muchas operaciones industriales. El valor del ángulo de contacto depende principalmente de la relación existente entre las fuerzas adhesivas del líquido y la superficie como de las internas de cohesión del propio líquido. Cuanta menor interacción hay, tanto más grande es el ángulo de contacto y su valor está relacionado directamente con la calidad de la limpieza, es decir, un menor ángulo de contacto presenta una mayor mojabilidad indicando una menor presencia de agentes contaminantes en la superficie de estudio y se considera que valores menores a $30^{\circ}$ presentan un alto grado de limpieza y que la superficie esta lista para recubrimientos posteriores. Para superficies rugosas el ángulo determinado aparente $\theta_{a p}$ y si la superficie tiene una inclinación el ángulo intrínseco $\theta_{i}[8,14,15]$ Figura 2.

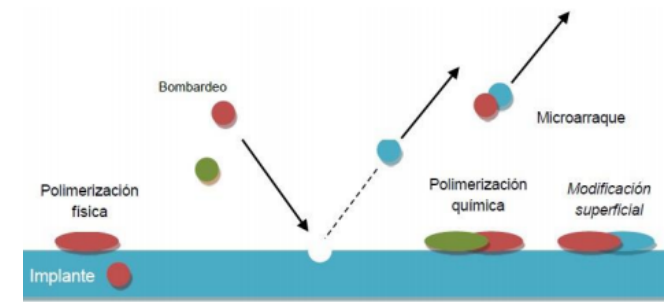

Figura 1. Efectos de modificación superficial del plasma. [16]

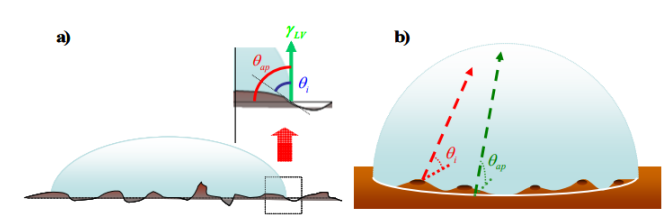

Figura 2. Ángulo de contacto intrínseco y aparente sobre una superficie. a) Rugosa y homogénea. b) Lisa y heterogénea. [15]

\section{Materiales y métodos}

\subsection{Materiales}

\subsubsection{Placas metálicas de acero inoxidable}

En la investigación se utilizaron láminas de acero inoxidable AISI/SAE 304 de 7 x $7 \mathrm{~cm}$ con un espesor de $2 \mathrm{~mm}$ para pruebas de laboratorio como se observa en la Figura 3, las mismas fueron impregnadas con $0,1 \mathrm{ml}$ de los aceites lubricantes ISO 32, ISO $68 \mathrm{y} \mathrm{el}$ ISO 220, previo a la descarga del plasma de oxígeno. No se consideró la rugosidad de la superficie del acero por no influir en el efecto que produce el gas ionizado de oxígeno sobre el volumen controlado de aceite depositado en la superficie.

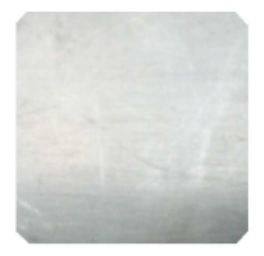

Figura 3. Placa de acero inoxidable. 


\subsubsection{Aceites lubricantes}

Los aceites lubricantes ISO 32, ISO 68 y el ISO 220 fueron utilizados como agentes contaminantes y colocados en la superficie de las láminas metálicas de acero inoxidable, los mismos fueron provistos por la casa comercial Gulf con las propiedades que se indican en la Tabla 1.

Tabla 1. Aceites lubricantes

\begin{tabular}{cccc}
\hline Aceite & $\begin{array}{c}\text { Viscosidad } \\
\mathbf{c S t} / \mathbf{4 0}{ }^{\circ} \mathbf{C}\end{array}$ & $\begin{array}{c}\text { Densidad a } \mathbf{~ 1 5}{ }^{\circ} \mathbf{C} \\
\mathbf{~ k g} / \mathbf{l}\end{array}$ & $\begin{array}{c}\text { Punto de } \\
\text { inflamabilidad }{ }^{\circ} \mathbf{C}\end{array}$ \\
\hline ISO 32 & 32 & 0,87 & 202 \\
ISO 68 & 68 & 0,88 & 218 \\
ISO 220 & 220 & 0,89 & 256 \\
\hline
\end{tabular}

\subsubsection{Oxígeno}

Para la generación del gas ionizado o plasma se utilizó oxígeno de alta pureza provisto por la empresa AGA. La presión de salida del tanque corresponde a 1 bar y su regulación se lo realizó en el equipo generador de plasma de acuerdo con las condiciones planteadas para la experimentación.

\subsubsection{Generador de plasma frío a baja presión}

El equipo generador de plasma utilizado es de marca Diener como se observa en la Figura 4, donde las partículas neutras e iones se suscitan entre los 25 a $100{ }^{\circ} \mathrm{C}$ con una temperatura electrónica entre los $105{ }^{\circ} \mathrm{C}$ a $5000{ }^{\circ} \mathrm{C}$ por medio de corriente continua y con presiones inferiores a los 133 mbar. El equipo es semiautomático donde se pueden utilizar tres gases diferentes para la generación del plasma y se pueden controlar los parámetros de presión, tiempo y potencia. [17].

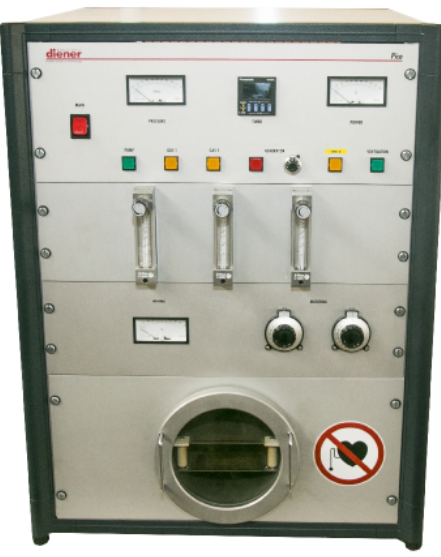

Figura 4. Generador de plasma.
En la Figura 5 se observa la cámara de plasma al vacío donde se colocaron las láminas de acero inoxidable impregnadas de aceite, además, se compone de una bomba de vacío de paletas rotativas de dos etapas que tiene una presión residual cercana a cero y permite la ventilación de la cámara.

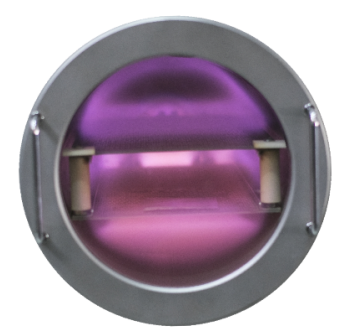

Figura 5. Cámara de plasma.

Los parámetros que se establecieron en el equipo generador de plasma de oxígeno se indican en la Tabla 2 , que según investigaciones realizadas por [8] son los idóneos en la limpieza de láminas metálicas con plasma frío de baja presión.

Tabla 2. Parámetros del generador de plasma

\begin{tabular}{ccccc}
\hline & \multicolumn{2}{c}{ Parámetros de control } & \\
Procesos & Lubricantes & $\begin{array}{c}\text { Tiempo } \\
\text { (min) }\end{array}$ & $\begin{array}{c}\text { Presión } \\
\text { (mbar) }\end{array}$ & $\begin{array}{c}\text { Potencia } \\
(\mathbf{\%})\end{array}$ \\
\hline Proceso & ISO 32 & 10 & 0,26 & 90 \\
con & ISO 68 & 10 & 0,30 & 90 \\
inmersión & ISO 220 & 15 & 0,32 & 90 \\
& & & & \\
Proceso & ISO 32 & 15 & 0,28 & 90 \\
sin & ISO 68 & 15 & 0,32 & 90 \\
inmersión & ISO 220 & 20 & 0,34 & 90 \\
\hline
\end{tabular}

\subsubsection{Analizador de gases}

El nivel de los gases generados en la limpieza de las láminas metálicas de acero inoxidable fueron detectados por un analizador de gas marca QROTECH modelo NGA 6000, como se observa en la Figura 6, tiene la capacidad para detectar monóxido de carbono en un rango de medición entre $0,00 \sim 0,99 \%$, que es altamente tóxico y puede ocasionar la muerte en niveles elevados, dióxido de carbono en un rango entre $0,0 \sim 20,0 \%$, que afecta al calentamiento global, oxígeno diatómico en un rango entre el $0,00 \sim 25,00$ $\%$, que no afecta al medioambiente, los hidrocarburos en un rango de medición entre $0 \sim 20000$ ppm, que son combinaciones de carbono e hidrógeno, comúnmente implicados en las intoxicaciones y los NOx en un rango entre $0 \sim 5000 \mathrm{ppm}$, que son reactivos como el óxido nítrico $(\mathrm{NO})$ y el dióxido de nitrógeno $\left(\mathrm{NO}_{2}\right)$ y muy perjudiciales para la salud, el ambiente y las estructuras. $[18,19]$ 


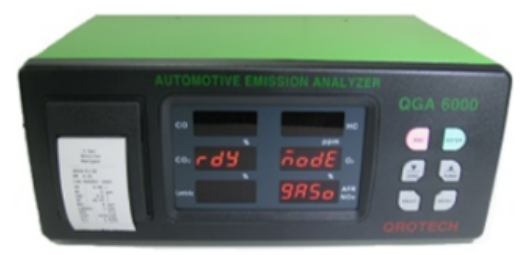

Figura 6. Analizador de gases.

\subsubsection{Goniómetro óptico digital}

El goniómetro óptico utilizado para determinar el ángulo de contacto del líquido de prueba sobre la superficie metálica es de marca KSV CAM100 como se observa en la Figura 7. Tiene incorporado una cámara CCD (charge-coupled device) con $50 \mathrm{~mm}$ de óptica y un software CAM 100 para el tratamiento de la imagen. Para la medición del ángulo de contacto se utilizó $5 \mu \mathrm{l}$ del líquido de prueba.

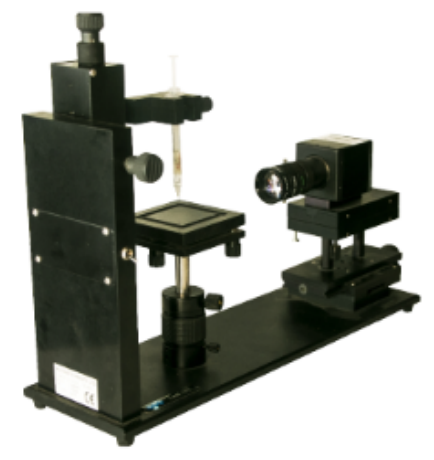

Figura 7. Goniómetro digital.

\subsubsection{Líquido de prueba}

Como líquido de prueba se utilizó agua desionizada o destilada (desmineralizada) con los componentes de la energía libre superficial que se presentan en la Tabla 3.

Tabla 3. Componentes del líquido de prueba. [20]

\begin{tabular}{lccc}
\hline $\begin{array}{c}\text { Líquido } \\
\text { de prueba }\end{array}$ & $\begin{array}{c}\text { Energía de } \\
\text { superficie }(\gamma)\end{array}$ & $\begin{array}{c}\text { Componente } \\
\text { dispersivo }\left(\gamma^{d}\right)\end{array}$ & $\begin{array}{c}\text { Componente } \\
\text { polar }\left(\gamma^{p}\right)\end{array}$ \\
\hline $\begin{array}{l}\text { Agua } \\
\text { desionizada }\end{array}$ & 72.80 & 21.80 & 51.00 \\
\hline
\end{tabular}

Los componentes del líquido de prueba obtenidos a través del ángulo de contacto con agua desionizada permite realizar el cálculo de la energía libre superficial mediante las teorías de Fowkes y Wu.

\subsection{Métodos}

\subsubsection{Método estadístico}

Para la investigación se utilizaron cinco muestras de láminas de acero inoxidable impregnadas con los aceites
ISO 32, ISO 68 e ISO 220 para el análisis en laboratorio donde los gases fueron detectados en partes por millón (ppm) y porcentaje (\%) como medidas de concentración, sin que influya la cantidad o volumen de aceite sobre la superficie. Para determinar si existe o no diferencia en los porcentajes y partes por millón de los gases generados en la limpieza por plasma frío a baja presión se aplicó un análisis de varianza (Anova) o una prueba de hipótesis para contrastar si las medias de los porcentajes del monóxido de carbono, dióxido de carbono y las partes por millón de los hidrocarburos son iguales con y sin inmersión en el desengrasante, donde posteriormente se analizó el gas que presentó la mayor contaminación.

$$
\begin{array}{ll}
H_{0}: \quad \mu_{1}=\mu_{2} & \begin{array}{l}
\text { No existe diferencia entre } \\
\text { los niveles de gases }
\end{array} \\
H_{1}: \quad \mu_{1} \neq \mu_{2} & \begin{array}{l}
\text { Existe diferencia entre los } \\
\text { niveles de gases }
\end{array}
\end{array}
$$

\subsubsection{Proceso}

La colocación del aceite contaminante para la impregnación sobre la superficie se lo realizó con una micropipeta. Los ensayos para determinar el nivel de contaminación de acuerdo con el grado de limpieza se hicieron con y sin inmersión en disolvente de la probeta previo a la aplicación de plasma frío como se indica en la Figura 8.

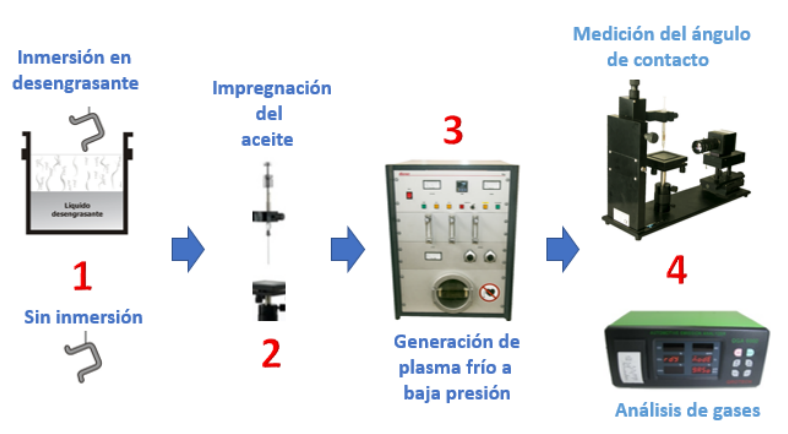

Figura 8. Procedimiento experimental.

Se realizó un predesengrase con una inmersión en un disolvente durante 3 minutos, de acuerdo con la cantidad de aceite a ser removido en la prueba de laboratorio. Para tratamientos industriales de limpieza para acero AISI/SAE 304 se recomienda de 30 a 60 minutos para eliminar compuestos orgánicos y remover los inorgánicos que pueden estar presentes en la superficie. La Tabla 4 presenta las propiedades del desengrasante utilizado. [21] 
López et al. / Análisis de la contaminación ambiental producida por el plasma frío de baja presión en la limpieza de láminas metálicas

Tabla 4. Propiedades del disolvente. [8]

\begin{tabular}{lc}
\hline Propiedad & Valor \\
\hline Densidad & $0,8 \mathrm{~g} / \mathrm{cm}^{3}$ \\
Temperatura de fusión & $-34^{\circ} \mathrm{C}$ \\
Temperatura de ebullición & $136^{\circ} \mathrm{C}$ \\
Temperatura de descomposición & $480^{\circ} \mathrm{C}$ \\
Inflamabilidad & $<37^{\circ} \mathrm{C}$ \\
\hline
\end{tabular}

\section{Resultados y discusión}

En las Tablas 5 y 6 se observan los niveles de gases obtenidos en la limpieza de las superficies de las láminas metálicas impregnadas con aceite ISO 32, aplicando plasma frío a baja presión.

Tabla 5. Valores del ángulo de contacto y niveles de gases con ISO 32 sin inmersión en desengrasante

\begin{tabular}{ccccccc}
\hline $\mathbf{N}$ & $\begin{array}{c}\text { Aceite } \\
(\mathbf{m l})\end{array}$ & $\begin{array}{c}\text { Ángulo de } \\
\text { contacto }\end{array}$ & $\begin{array}{c}\text { Líquido de } \\
\text { prueba }(\mu \mathbf{l})\end{array}$ & $\begin{array}{c}\mathbf{C O} \\
(\%)\end{array}$ & $\begin{array}{c}\mathbf{C O}_{2} \\
(\%)\end{array}$ & $\begin{array}{c}\mathbf{H C} \\
(\mathbf{p p m})\end{array}$ \\
\hline 1 & & $16,76^{\circ}$ & & 0,34 & 0,1 & 563 \\
2 & & $16,49^{\circ}$ & & 0,38 & 0,1 & 576 \\
3 & 0,1 & $17,14^{\circ}$ & 5 & 0,37 & 0,1 & 553 \\
4 & & $16,24^{\circ}$ & & 0,38 & 0,1 & 585 \\
5 & & $17,32^{\circ}$ & & 0,41 & 0,1 & 601 \\
\hline
\end{tabular}

Tabla 6. Valores del ángulo de contacto y niveles de gases con ISO 32 con inmersión en desengrasante

\begin{tabular}{ccccccc}
\hline $\mathbf{N}$ & $\begin{array}{c}\text { Aceite } \\
(\mathbf{m l})\end{array}$ & $\begin{array}{c}\text { Ángulo de } \\
\text { contacto }\end{array}$ & $\begin{array}{c}\text { Líquido de } \\
\text { prueba }(\mu \mathbf{l})\end{array}$ & $\begin{array}{c}\mathbf{C O} \\
(\mathbf{\%})\end{array}$ & $\begin{array}{c}\mathbf{C O}_{2} \\
(\mathbf{\%})\end{array}$ & $\begin{array}{c}\mathbf{H C} \\
(\mathbf{p p m})\end{array}$ \\
\hline 1 & & $12,92^{\circ}$ & & 0,34 & 0,1 & 1031 \\
2 & & $12,25^{\circ}$ & & 0,33 & 0,1 & 1028 \\
3 & 0,1 & $12,73^{\circ}$ & 5 & 0,34 & 0,1 & 1049 \\
4 & & $12,86^{\circ}$ & & 0,34 & 0,1 & 995 \\
5 & & $12,27^{\circ}$ & & 0,37 & 0,1 & 1026 \\
\hline
\end{tabular}

Para determinar si existe una diferencia entre los niveles de contaminación de los gases obtenidos en los procesos con y sin inmersión, en el desengrasante se realizó un análisis de varianzas (Anova) entre los grupos de gases, monóxido de carbono e hidrocarburos. Se planteó como hipótesis nula que no existe diferencia entre los grupos analizados con un nivel de significación $\alpha=0.05$, obteniendo los resultados que se presentan a continuación en las Tablas 7 y 8.

Tabla 7. ANOVA de un factor. ISO 32 (CO)

\begin{tabular}{|c|c|c|c|c|c|}
\hline & $\begin{array}{c}\text { Suma de } \\
\text { cuadrados }\end{array}$ & gl & $\begin{array}{c}\text { Media } \\
\text { cuadrática }\end{array}$ & F & Sig. \\
\hline Inter-grupos & 0,003 & 1 & 0,003 & 5,953 & 0,041 \\
\hline Intra-grupos & 0,003 & 8 & 0,000 & & \\
\hline Total & 0,006 & 9 & & & \\
\hline
\end{tabular}

Tabla 8. ANOVA de un factor. ISO 32 (HC)

\begin{tabular}{lrrrrr} 
ppm de HC & \multicolumn{1}{c}{ Suma de } & Media & F & Sig. \\
& cuadrados & gl & cuadrática & F & \\
\hline Inter-grupos & 506700,1 & 1 & 506700,10 & 1387,269 & 0,000 \\
Intra-grupos & 2922,0 & 8 & 365,25 & & \\
Total & 509622,1 & 9 & & & \\
\hline
\end{tabular}

Como se observa en las Tablas 7 y 8 el valor de p (Sig.) es menor a 0,05 lo que indicó una diferencia entre los porcentajes de monóxido de carbono y los hidrocarburos en los dos procesos con y sin inmersión en desengrasante, determinándose que existe una mayor contaminación al realizar una inmersión previa, sin embargo, también presenta una mejor limpieza como se observa en los valores del ángulo de contacto indicados en las Tablas 5 y 6 . Los niveles de dióxido de carbono permanecieron constantes en los procesos con y sin inmersión.

En las Tablas 9 y 10 se observan los niveles de gases obtenidos en la limpieza de la superficie de las láminas metálicas de acero inoxidable impregnadas con el aceite ISO 68.

Tabla 9. Valores del ángulo de contacto y niveles de gases. ISO 68 sin inmersión en desengrasante.

\begin{tabular}{ccccccc}
\hline $\mathbf{N}$ & $\begin{array}{c}\text { Aceite } \\
(\mathbf{m l})\end{array}$ & $\begin{array}{c}\text { Ángulo de } \\
\text { contacto }\end{array}$ & $\begin{array}{c}\text { Líquido de } \\
\text { prueba }(\mu \mathbf{l})\end{array}$ & $\begin{array}{c}\mathbf{C O} \\
(\boldsymbol{\%})\end{array}$ & $\begin{array}{c}\mathbf{C O}_{2} \\
(\%)\end{array}$ & $\begin{array}{c}\mathbf{H C} \\
(\mathbf{p p m})\end{array}$ \\
\hline 1 & & $36,93^{\circ}$ & & 0,50 & 0,2 & 1177 \\
2 & & $37,55^{\circ}$ & & 0,50 & 0,2 & 1151 \\
3 & 0,1 & $36,86^{\circ}$ & 5 & 0,48 & 0,2 & 1098 \\
4 & & $36,91^{\circ}$ & & 0,49 & 0,2 & 1133 \\
5 & & $36,05^{\circ}$ & & 0,37 & 0,2 & 1088 \\
\hline
\end{tabular}

Tabla 10. Valores del ángulo de contacto y niveles de gases. ISO 68 con inmersión en desengrasante.

\begin{tabular}{ccccccc}
\hline $\mathbf{N}$ & $\begin{array}{c}\text { Aceite } \\
(\mathbf{m l})\end{array}$ & $\begin{array}{c}\text { Ángulo de } \\
\text { contacto }\end{array}$ & $\begin{array}{c}\text { Líquido de } \\
\text { prueba }(\mu \mathbf{l})\end{array}$ & $\begin{array}{c}\mathbf{C O} \\
(\%)\end{array}$ & $\begin{array}{c}\mathbf{C O}_{2} \\
(\%)\end{array}$ & $\begin{array}{c}\mathbf{H C} \\
(\mathbf{p p m})\end{array}$ \\
\hline 1 & & $16,03^{\circ}$ & & 0,39 & 0,2 & 2068 \\
2 & & $16,52^{\circ}$ & & 0,45 & 0,2 & 2214 \\
3 & 0,1 & $16,35^{\circ}$ & 5 & 0,44 & 0,2 & 2315 \\
4 & & $16,96^{\circ}$ & & 0,49 & 0,2 & 2185 \\
5 & & $16,83^{\circ}$ & & 0,50 & 0,2 & 2220 \\
\hline
\end{tabular}

Para determinar si existe o no una diferencia entre los niveles de contaminación de los gases generados en la limpieza de la superficie de las láminas metálicas de acero inoxidable impregnadas con el aceite ISO 68 en los procesos con y sin inmersión en un líquido desengrasante, se aplicó un análisis con un nivel de confianza del $95 \%$. Las Tablas 11 y 12 presentan los resultados obtenidos.

Tabla 11. ANOVA de un factor. ISO 68 (CO)

\begin{tabular}{|c|c|c|c|c|c|}
\hline & $\begin{array}{c}\text { Suma de } \\
\text { cuadrados }\end{array}$ & gl & $\begin{array}{c}\text { Media } \\
\text { cuadrática }\end{array}$ & $\mathbf{F}$ & Sig. \\
\hline Inter-grupos & 0,000 & 1 & 0,000 & 0,196 & 0,670 \\
\hline Intra-grupos & 0,020 & 8 & 0,003 & & \\
\hline Total & 0,020 & 9 & & & \\
\hline
\end{tabular}


Tabla 12. ANOVA de un factor. ISO 68 (HC)

\begin{tabular}{lrrrrr} 
ppm de HC & $\begin{array}{c}\text { Suma de } \\
\text { cuadrados }\end{array}$ & gl & $\begin{array}{c}\text { Media } \\
\text { cuadrática }\end{array}$ & F & Sig. \\
\hline Inter-grupos & 286702,5 & 1 & 2867602,50 & 621,460 & 0,000 \\
Intra-grupos & 36914,4 & 8 & 4614,30 & & \\
Total & 2904516,9 & 9 & & & \\
\hline
\end{tabular}

El resultado de la Tabla 11 presentó un valor de p (Sig.) de 0,670 >0,05, por lo tanto, los niveles de monóxido de carbono son los mismos en los procesos con y sin inmersión en desengrasante. La Tabla 12 mostró un valor $\mathrm{p}$ (Sig.) de $0,000<0,05$ lo que indicó que los niveles de los hidrocarburos son diferentes, dando un incremento cuando se realiza una inmersión previa en desengrasante y los niveles de dióxido de carbono permanecieron constantes como se observan en las Tablas 9 y 10.

Los niveles de gases obtenidos en la limpieza con plasma frío a baja presión de las superficies de láminas metálicas de acero inoxidable impregnadas con aceite ISO 220 en los procesos con y sin inmersión previa en un líquido desengrasante, se observan en las Tablas 13 y 14.

Tabla 13. Valores del ángulo de contacto y niveles de gases. ISO 220 sin inmersión en desengrasante

\begin{tabular}{ccccccc}
\hline $\mathbf{N}$ & $\begin{array}{c}\text { Aceite } \\
(\mathbf{m l})\end{array}$ & $\begin{array}{c}\text { Ángulo de } \\
\text { contacto }\end{array}$ & $\begin{array}{c}\text { Líquido de } \\
\text { prueba }(\mu \mathbf{l})\end{array}$ & $\begin{array}{c}\mathbf{C O} \\
(\mathbf{\%})\end{array}$ & $\begin{array}{c}\mathbf{C O}_{2} \\
(\mathbf{\%})\end{array}$ & $\begin{array}{c}\mathbf{H C} \\
(\mathbf{p p m})\end{array}$ \\
\hline 1 & & $71,41^{\circ}$ & & 0,47 & 0,2 & 1259 \\
2 & & $71,49^{\circ}$ & & 0,45 & 0,2 & 1317 \\
3 & 0,1 & $71,16^{\circ}$ & 5 & 0,47 & 0,2 & 1199 \\
4 & & $71,94^{\circ}$ & & 0,49 & 0,2 & 1278 \\
5 & & $71,32^{\circ}$ & & 0,50 & 0,2 & 1344 \\
\hline
\end{tabular}

Tabla 14. Valores del ángulo de contacto y niveles de gases. ISO 220 con inmersión en desengrasante

\begin{tabular}{ccccccc}
\hline $\mathbf{N}$ & $\begin{array}{c}\text { Aceite } \\
(\mathbf{m l})\end{array}$ & $\begin{array}{c}\text { Ángulo de } \\
\text { contacto }\end{array}$ & $\begin{array}{c}\text { Líquido de } \\
\text { prueba }(\mu \mathbf{l})\end{array}$ & $\begin{array}{c}\mathbf{C O} \\
(\mathbf{\%})\end{array}$ & $\begin{array}{c}\mathbf{C O}_{2} \\
(\boldsymbol{\%})\end{array}$ & $\begin{array}{c}\mathbf{H C} \\
(\mathbf{p p m})\end{array}$ \\
\hline 1 & & $20,50^{\circ}$ & & 0,51 & 0,4 & 1885 \\
2 & & $20,69^{\circ}$ & & 0,52 & 0,3 & 1902 \\
3 & 0,1 & $20,93^{\circ}$ & 5 & 0,51 & 0,4 & 1925 \\
4 & & $20,80^{\circ}$ & & 0,50 & 0,4 & 1975 \\
5 & & $20,00^{\circ}$ & & 0,51 & 0,4 & 1932 \\
\hline
\end{tabular}

Para determinar si existe una diferencia entre los niveles de los gases generados en la limpieza de la superficie de las láminas metálicas de acero inoxidable impregnadas con el aceite ISO 220 con y sin inmersión en desengrasante, se aplicó un análisis Anova obteniendo los datos que se indican en las Tablas $15 \mathrm{y}$ 16.

Tabla 15. ANOVA de un factor. ISO 220 (CO)

Porcentaje de CO

\begin{tabular}{lccccc}
\hline & $\begin{array}{c}\text { Suma de } \\
\text { cuadrados }\end{array}$ & gl & $\begin{array}{c}\text { Media } \\
\text { cuadrática }\end{array}$ & F & Sig. \\
\hline Inter-grupos & 0,003 & 1 & 0,003 & 13,442 & 0,006 \\
Intra-grupos & 0,002 & 8 & 0,000 & & \\
Total & 0,005 & 9 & & & \\
\hline
\end{tabular}

Tabla 16. ANOVA de un factor. ISO 220 (HC)

\begin{tabular}{lrrr|cr} 
ppm de HC & $\begin{array}{c}\text { Suma de } \\
\text { cuadrados }\end{array}$ & gl & $\begin{array}{c}\text { Media } \\
\text { cuadrática }\end{array}$ & F & Sig. \\
\hline Inter-grupos & 1038128,4 & 1 & 1038128,40 & 484,541 & 0,000 \\
Intra-grupos & 17140,0 & 8 & 2142,50 & & \\
Total & 1055268,4 & 9 & & & \\
\hline
\end{tabular}

Las Tablas 15 y 16 indican valores de p (Sig.) menores a 0,05 por lo que se afirma una diferencia en los niveles de contaminación al realizar la limpieza de la superficie de la lámina metálica impregnada con el aceite ISO 220, sin y con una inmersión previa en desengrasante antes de aplicar el plasma frío a baja presión.

La Tabla 17 presenta los promedios de los resultados obtenidos en los niveles de contaminación de los gases generados al aplicar un plasma de oxígeno en la limpieza de láminas metálicas.

Tabla 17. Resumen de los valores promedio de ángulo de contacto y nivel de gases generados

\begin{tabular}{|c|c|c|c|}
\hline & ISO 32 & ISO 68 & ISO 220 \\
\hline \multicolumn{4}{|l|}{ CON INMERSIÓN } \\
\hline Ángulo de contacto & $12,606^{\circ}$ & $16.538^{\circ}$ & $20,584^{\circ}$ \\
\hline Porcentaje de CO & 0,344 & 0,454 & 0,510 \\
\hline Porcentaje de $\mathrm{CO}_{2}$ & 0,1 & 0,2 & 0,38 \\
\hline Partes por millón de $\mathrm{HC}$ & 1025,8 & 2200,4 & 1923,8 \\
\hline \multicolumn{4}{|l|}{ SIN INMERSIÓN } \\
\hline Ángulo de contacto & $16,79^{\circ}$ & $36,46^{\circ}$ & $71,464^{\circ}$ \\
\hline Porcentaje de CO & 0,376 & 0,468 & 0,476 \\
\hline Porcentaje de $\mathrm{CO}_{2}$ & 0,1 & 0,2 & 0,2 \\
\hline Partes por millón de $\mathrm{HC}$ & 575,6 & 1129,4 & 1279,4 \\
\hline
\end{tabular}

\section{Conclusiones}

Los procesos de limpieza en láminas de acero inoxidable AISI/SAE 304 con plasma frío a baja presión sin y con inmersión en un desengrasante previo a la aplicación del gas ionizado presentan diferencias en los niveles de los gases desprendidos. De acuerdo con los promedios de los resultados, en la remoción del aceite ISO 32 de la superficie con el plasma frío a baja presión, la inmersión previa en el desengrasante aporta a disminuir el ángulo de contacto en un $25 \%$, lo que indica una mejor calidad de limpieza en la superficie, sin embargo, los niveles de contaminación sufren una variación, en especial los hidrocarburos al aumentar considerablemente en un 78,21\% debido a que remueve el aceite de la superficie junto con restos del desengrasante, el nivel de monóxido de carbono disminuye en un $8,5 \%$ y el dióxido de carbono permanece constante, lo que sugiere que para la remoción del aceite ISO 32 no es necesario una inmersión previa ya que sin ella se obtiene un ángulo de contacto óptimo $\left(\right.$ menor a $\left.30^{\circ}\right)$ y genera una menor contaminación. 
En la remoción del aceite ISO 68 se observó que con una inmersión de las láminas metálicas en desengrasante antes de aplicar el proceso de limpieza por plasma frío a baja presión, que el nivel de los hidrocarburos aumenta en un $95 \%$, el porcentaje del monóxido de carbono disminuye en un $3 \%$ y el porcentaje de dióxido de carbono permanece constante con respecto a los valores obtenidos sin una inmersión previa. El ángulo de contacto disminuye en un $54 \%$ con una inmersión previa en desengrasante antes de la descarga de plasma, obteniendo valores óptimos de limpieza menores a $30^{\circ}$.

En la remoción del aceite ISO 220 de la superficie se observó un aumento en el nivel de contaminación en todos los gases generados en la limpieza de láminas metálicas con plasma frío a baja presión cuando se aplicó una inmersión previa en desengrasante para disminuir el ángulo de contacto en un $71 \%$ con respecto al proceso sin inmersión. Los hidrocarburos aumentaron en un $50 \%$ sus partes por millón, el porcentaje del monóxido de carbono aumentó en un $7 \%$ y el dióxido de carbono en un $90 \%$.

En la limpieza de las superficies de las láminas metálicas, el aceite ISO 68 presenta la mayor contaminación promedio en hidrocarburos con 2200,4 ppm a un ángulo de contacto promedio de $16,53^{\circ}$. El aceite ISO 220 presenta una mayor contaminación promedio de monóxido de carbono al $0,51 \%$ y de dióxido de carbono al $0,38 \%$.

\section{Referencias}

[1] C. E. Fúquene Retamoso, Producción limpia, contaminación y gestión ambiental. Editorial Pontificia Universidad Javeriana, 2007. [Online]. Available: http://bit.ly/2YNLtud

[2] C. Frías and O. Pérez, "Recuperación de ácidos y metales en baños agotados del decapado de aceros inoxidables," Revista de Metalurgia, vol. 34, pp. 427-431, 1998. [Online]. Available: https://doi. org/10.3989/revmetalm.1998.v34.iExtra.786

[3] M. Villalobos, D. Peñalosa, M. Moscona, M. Cram, and F. Heydrich, "Opciones para el tratamiento y la disposición de los licores ácidos agotados provenientes del decapado de hierro," Revista Internacional de contaminación ambiental, vol. 6, no. 1, pp. 33-54, 1990. [Online]. Available: http://bit.ly/2WVmXa0

[4] E. Cano Díaz, J. Simancas Peco, L. Narváez, and J. M. Bastidas Rull, Estudio de la corrosión del cobre por vapores de ácido acético al 40 y $80 \%$ de humedad relativa. Sociedad Española de Cerámica y Vidrio, 2004. [Online]. Available: http://bit.ly/2M48IOX
[5] N. Ipek, N. Lior, and A. Eklund, "Improvement of the electrolytic metal pickling process by inter-electrode insulation," Ironmaking \& Steelmaking. Processes, Products and Applications, vol. 32, no. 1, pp. 87-96, 2005. [Online]. Available: https://doi.org/10.1179/174328105X23996

[6] R. d'Agostino, P. Favia, C. Oehr, and M. R. Wertheimer, "Low-temperature plasma processing of materials: Past, present, and future," Plasma Processes and Polymers, vol. 2, no. 1, pp. 7-15, 2005. [Online]. Available: https://doi.org/10.1002/ppap.200400074

[7] R. Nickerson, "Plasma surface modification for cleaning and adhesion," AST Products, Inc, vol. Billerica, MA. 01821, 1998.

[8] P. Sarmiento, L. López, A. Sarmiento, and J. Fajardo, "Efficiency of the low pressure cold plasma in the cleaning of steel for subsequent covering," VI Andean Region International Conference (ANDESCOM), vol. 5, pp. 115-118, 2012. [Online]. Available: https://doi.org/10.1109/Andescon.2012.35

[9] D. Y. Kwok and A. W. Neuman, "Contact angle measurement and contact angle interpretation," Advances in Colloid and Interface Science, vol. 81, no. 3, pp. 167-249, 1999. [Online]. Available: https://doi.org/10.1016/S0001-8686(98)00087-6

[10] D. O. Njobuenwu, E. O. Oboho, and R. H. Gumus, "Determination of contact angle from contact area of liquid droplet spreading on solid substrate," Electronic Journal of Practices and Technologies, vol. 6, no. 10, pp. 29-38, 2007. [Online]. Available: http://bit.ly/2M34F5K

[11] M. Zenkiewicz, "Methods for the calculation of surface free energy of solids," Journal of Achievements in Materials and Manufacturing Engineering, vol. 24, no. 1, pp. 137-145, 2007. [Online]. Available: http://bit.ly/2ErRPYn

[12] J. R. Roth, Industrial Plasma Engineering. CRC press. Taylor Francis Group, 2001, vol. 2. [Online]. Available: http://bit.ly/2EoRPZi

[13] F. Gordillo, Plasmas fríos. Investigación y Ciencia, 2008. [Online]. Available: http: //bit.ly/2HQqZtV

[14] G. Neira Arenas and L. A. Cañas M, "Procedimiento para medir ángulos de contacto en sólidos particulados finos," Scientia et Technica, vol. 1, no. 36 , pp. $883-887,2007$. [Online]. Available: http://dx.doi.org/10.22517/23447214.5159 
[15] J. M. Ruiz-Cabello, "Efecto de la rugosidad y heterogeneidad superficial en fenómenos de mojado," Ph.D. dissertation, Universidad de Granada, 2009. [Online]. Available: http://bit.ly/2VJYgvu

[16] E. Fuster, "Aplicación de plasma atmosférico en tratamiento superficial de metales para utilización de adhesivos estructurales," Master's thesis, Universidad Politécnica de Valencia, 2016. [Online]. Available: http://bit.ly/2JzOxq5

[17] Diener, Introducción al plasma. Diener electronic. Plasma-Surface-Technology, 2016. [Online]. Available: http://bit.ly/2M1zs2C

[18] D. Galán and R. Fernández, "Implicación de los nox en la química atmosférica," Revista electrónica de medioambiente UCM, vol. 2, pp. 90-103, 2006. [Online]. Available: http://bit.ly/2.JDI8KC
[19] J. D. Escrig Zaragozá, El impacto ambiental de las actividades industriales: el cambio necesario. Universidad Internacional de Andalucía, España, 2008. [Online]. Available: http://bit.ly/2EtFjrv

[20] Z. Navrátil, V. Buršíková, P. St'ahel, M. Š́ra, and P. Zvěrina, "On the analysis or surface fre energy of DLC costings deposited in low pressure RF discharge," Czechoslovak Journal of Physics, vol. 54, no. 3, pp. 29-38, C877 2004. [Online]. Available: https://doi.org/10.1007/BF03166502

[21] Grupo Böhler, Libro del decapado. Tratamientos superficiales del acero inoxidable. Grupo Böhler Soldadura España, 2017. [Online]. Available: http://bit.ly/2EsgJXL 